\title{
The prognostic value of quality of life scores during treatment for oesophageal cancer
}

\author{
J M Blazeby, S T Brookes, D Alderson
}

\begin{abstract}
Background-Quality of life (QL) data are useful to evaluate the effectiveness of treatment. Accumulating evidence suggests that QL data may predict survival.

Aims-In this study we investigated if baseline QL scores and changes in $Q L$ scores before and after intervention are prognostic for patients with oesophageal cancer.

Patients-Between 1993 and 1995, 92 consecutive new patients with oesophageal cancer were studied; 89 were followed until death or the end of the study period (survival of seven patients was censored in May 1999).

Methods-All patients completed the European Organisation for Research and Treatment of Cancer (EORTC) core questionnaire (EORTC QLQ-C30) and the dysphagia scale of the oesophageal module (EORTC QLQ-OES24) before treatment and at regular intervals throughout the study. Cox's proportional hazards models assessed the impact of baseline QL variables and changes in $Q L$ scores on survival.
\end{abstract}

Results-Cox's proportional hazards models, adjusting for associations between QL scores, age, and TNM stage, found that physical function at baseline was significantly associated with survival $(p=0.002)$. An increase in physical function score of 10 points corresponded to a $12 \%$ reduction in the likelihood of death at any given time $(95 \%$ confidence intervals 4-18\%). Further exploratory multivariable analyses suggested that improvement in emotional function six months after treatment was significantly related to longer survival $(\mathrm{p}<0.0001)$.

Division of Surgery, Bristol Royal Infirmary, Bristol BS2 8HW, UK J M Blazeby D Alderson

Department of Social Medicine, University of Bristol, Bristol BS8 2PR, UK

$\mathrm{S}$ T Brookes

Correspondence to: Miss J M Blazeby, Division of Surgery, Bristol BS2 8HW, UK. jmblazeby@hotmail.com

Accepted for publication 29 January 2001

Accurate assessment of quality of life (QL) in patients with cancer is important because it provides detailed information about patients' perceptions of their health. Such QL data can be used to decide the relative effectiveness of treatment, enhance patients' decision making by providing more information on the side effects of treatment, and inform economic analyses for the organisation of cancer services. Evidence is now accumulating to indicate that QL scores can predict survival. ${ }^{1-3}$ In patients with metastatic lung cancer, overall QL scores were associated with survival, independent of disease stage and cell type. ${ }^{4}$ In advanced breast cancer, good physical function and high overall patient rated health scores at diagnosis were powerful independent predictors of survival, and in patients with metastatic melanoma, assessments of mood, appetite loss, and overall QL have been shown to significantly predict survival, independent of established prognostic factors. ${ }^{56}$ Others have found that in advanced breast cancer, high dyspnoea scores were prognostic for survival. ${ }^{7}$ Changes in QL scores after chemotherapy for breast cancer have also been found to be prognostically important, where improvements in physical well being, mood, and pain scores significantly predicted longer survival.

Several studies have examined QL in patients with oesophageal cancer but the prognostic value of valid QL scores in this patient group has not been investigated. ${ }^{8-10}$ Identification of prognostic factors in patients with oesophageal cancer is important because this may modify management decisions regarding curative or palliative treatment. The aim of this study was to investigate if baseline QL scores and changes in QL scores during treatment are related to survival in patients with carcinoma of the oesophagus.

\section{Methods}

Between November 1993 and May 1995 baseline clinical data of consecutive new patients presenting with carcinoma of the oesophagus to the Bristol Royal Infirmary and Frenchay Hospital, Bristol, UK, were prospectively entered into a database. Standard assessments of general health and tumour stage included full blood count, blood chemistry, electrocardiography, pulmonary function tests, chest radiography, endoscopy, and biopsy. Computed tomography and/or magnetic resonance imaging were performed to exclude distant metastases. Patients without major co-morbid disease or evidence of distant haematogenous disease spread underwent oesophagectomy.
Abbreviations used in this paper: QL, quality of life; EORTC, European Organisation for Research and Treatment of Cancer. 
Patients unfit for oesophagectomy or with distant metastatic disease were treated by intubation or palliative chemoradiotherapy. All patients were followed until death or the end of the study period (May 1999) when all surviving patients were censored. Informed consent and ethics committee permission were obtained.

QUALITY OF LIFE ASSESSMENT

QL was assessed at baseline 1-2 weeks before treatment using the European Organisation for Research and Treatment of Cancer core QL questionnaire (EORTC QLQ-C30, version 1.0) and the dysphagia scale from the oesophageal cancer module (EORTC QLQOES24). ${ }^{112}$ Further QL assessments were performed 4-6 weeks after oesophagectomy, every three months during the first postoperative year, every six months during the second year, and annually thereafter. After treatment with palliative intent, assessments were performed monthly. Data were collected until death or for a minimum of three postoperative years. Baseline and first postoperative assessments of patients undergoing oesophagectomy were performed in the patients' homes. Subsequent questionnaires were mailed unless additional help was required. All patients receiving palliative treatment were visited at home for every assessment. Questionnaires were checked for omitted answers before completion of the interviews; postal questionnaires were checked for missing data. If answers were absent, patients were contacted and asked the omitted questions. The EORTC QLQ-C30 is a valid and reliable measure of QL which has been tested in patients with cancer of the oesophagus. ${ }^{13}$ It includes five functional scales (physical, role, emotional, cognitive, and social), three symptom scales (fatigue, pain, and emesis), and one global scale. Five single items are designed to assess additional symptoms (dyspnoea, sleep disturbance, appetite loss, constipation, and diarrhoea). The last item is related to the perceived financial impact of cancer and its treatment. Only the dysphagia scale from the EORTC QLQ-OES24 was used because at the time of this study the questionnaire was not fully developed. QL scores were calculated according to standard guidelines, yielding a range of $0-100 .{ }^{14} \mathrm{High}$ scores in the function and global QL scales represent good QL whereas high symptom scores reflect worse QL.

STATISTICAL ANALYSES

Cox's proportional hazards models were used to assess the impact of QL on survival. ${ }^{15}$ In the first instance univariable analyses were performed, examining the effect on survival of each baseline QL score in turn. Multivariable models were then generated to control for possible confounding effects of established prognostic factors and associations between QL scores. Established prognostic factors that were adjusted for were patient age, and $\mathrm{T}, \mathrm{N}$, and $\mathrm{M}$ stage. Additional possible prognostic factors examined included sex, tumour type, ASA grade, and treatment group. A nominal significance level of $1 \%$ was used throughout.

Six months after treatment, changes between baseline and six month QL scores were calculated for all surviving patients. Cox's proportional hazards models were used to investigate whether changes in QL scores following treatment were related to survival. Because of the reduced sample size of surviving patients at six months, this secondary analysis was intended as a purely hypothesis generating exercise.

\section{Results}

Ninety two new patients presented during the study period; 55 underwent oesophagectomy, 30 were intubated, and seven patients underwent primary palliative radiotherapy or chemotherapy. Three patients (who underwent oesophagectomy) were excluded because QL data were not obtained before the start of treatment. Clinical characteristics of the 89 patients who completed baseline assessments are shown in table 1.

Nine patients died within 30 days of surgery and another 17 experienced major complications. Seven patients experienced morbidity after palliation of malignant dysphagia, of whom five died within 30 days of treatment. At six months after treatment, 51 patients had died. Of the 38 patients alive six months after treatment, 34 had undergone oesophagectomy and four endoscopic palliation. Clinical characteristics of the 38 patients who completed six month assessments are shown in table 1. At the end of follow up (May 1999), the seven alive patients were censored. Median survival time after oesophagectomy and palliative treatment was 11.5 and 2.5 months, respectively. The descriptive results of the longitudinal QL data have been published elsewhere. ${ }^{16}$

Median baseline QL scores are shown in table 2. Cox's proportional hazards models, adjusting for age, and $\mathrm{T}, \mathrm{N}$, and $\mathrm{M}$ stage, found that better physical function score was significantly associated with longer survival $(p=0.002)$, and worse fatigue was significantly associated with shorter survival $(p=0.001)$ (table 3). However, when adjusting for associations between QL scores (by including more than one QL score in the model), only physical function at baseline remained significantly associated with survival. The final model is

Table 1 Patient details

\begin{tabular}{lcl}
\hline & $\begin{array}{l}\text { All patients } \\
(n=89)\end{array}$ & $\begin{array}{l}\text { Patients alive 6 } \\
\text { months after } \\
\text { treatment }(n=38)\end{array}$ \\
\hline Age (y) & $70(41-94)$ & $65(41-94)$ \\
Sex (male/female) & $55 / 34$ & $21 / 17$ \\
Histology (adeno/squamous) & $52 / 37$ & $21 / 17$ \\
Tumour length (cm) & $5(2-15)$ & $6(2-12)$ \\
Distance incisors (cm) & $36(22-40)$ & $34(22-40)$ \\
Pathological stage ${ }^{20}$ & & \\
$\quad$ Stage I & 0 & 0 \\
Stage IIA & 11 & 10 \\
Stage IIB & 4 & 3 \\
Stage III & 42 & 21 \\
Stage IV & 25 & 1 \\
Incomplete staging data & 7 & 3 \\
\hline
\end{tabular}

Values are median (range) or number. 
Table 2 Baseline and six month median quality of life (QL) scores (median (interquartile range))

\begin{tabular}{lcc}
\hline QL variable & $\begin{array}{c}\text { Baseline } Q L \text { score } \\
(n=89)\end{array}$ & $\begin{array}{l}6 \text { month } Q L \text { score } \\
(n=38)\end{array}$ \\
\hline Physical & $80(40-100)$ & $60(40-100)$ \\
Role & $100(50-100)$ & $50(50-100)$ \\
Social & $83(50-100)$ & $67(33-100)$ \\
Cognitive & $83(83-100)$ & $83(67-100)$ \\
Emotional & $83(67-100)$ & $83(58-100)$ \\
Global QL & $50(33-75)$ & $54(42-67)$ \\
Fatigue & $33(11-56)$ & $33(22-56)$ \\
Anorexia & $33(0-67)$ & $33(0-67)$ \\
Emesis & $17(0-33)$ & $0(0-33)$ \\
Constipation & $33(0-33)$ & $0(0-33)$ \\
Diarrhoea & $0(0-0)$ & $0(0-33)$ \\
Pain & $17(0-33)$ & $17(0-33)$ \\
Dyspnoea & $0(0-33)$ & $33(0-33)$ \\
Sleep problems & $0(0-33)$ & $17(0-33)$ \\
Finance problems & $0(0-0)$ & $0(0-0)$ \\
Dysphagia & $44(22-67)$ & $22(0-33)$ \\
\hline
\end{tabular}

Table 3 Cox's proportional hazards models adjusting for age, and $T, N$, and $M$ stage and considering each baseline quality of life $(Q L)$ score in turn $(n=89)$

\begin{tabular}{llll}
\hline QL variable & $\begin{array}{c}\text { Hazard } \\
\text { ratio }\end{array}$ & $95 \%$ CI & p Value \\
\hline Physical & 0.88 & $0.82-0.96$ & 0.002 \\
Role & 0.93 & $0.87-0.99$ & 0.032 \\
Social & 0.91 & $0.84-0.99$ & 0.028 \\
Cognitive & 0.93 & $0.84-1.03$ & 0.161 \\
Emotional & 1.05 & $0.94-1.17$ & 0.420 \\
Global QL & 0.96 & $0.87-1.06$ & 0.419 \\
Fatigue & 1.15 & $1.06-1.25$ & 0.001 \\
Anorexia & 1.05 & $0.99-1.12$ & 0.100 \\
Emesis & 1.03 & $0.94-1.13$ & 0.502 \\
Constipation & 1.11 & $1.02-1.21$ & 0.020 \\
Diarrhoea & 0.96 & $0.83-1.12$ & 0.615 \\
Pain & 1.11 & $1.02-1.22$ & 0.017 \\
Dyspnoea & 1.06 & $0.99-1.15$ & 0.108 \\
Sleep problems & 1.00 & $0.93-1.08$ & 0.933 \\
Finance problems & 1.12 & $0.97-1.30$ & 0.122 \\
Dysphagia & 1.07 & $0.97-1.17$ & 0.161 \\
\hline
\end{tabular}

^Hazard ratio provides the likelihood of death at any given time for a patient with a score of 10 points more than another patient.

Table 4 Multivariable analyses of baseline quality of life (QL) scores, $n=89$. The final model

\begin{tabular}{llll}
\hline Variable & $\begin{array}{l}\text { Hazard } \\
\text { ratio }\end{array}$ & $95 \%$ CI & p Value \\
\hline Age & $1.38^{\star}$ & $1.10-0.96$ & 0.005 \\
T3 $v$ T2 stage & 0.86 & $0.33-2.26$ & $0.801 \dagger$ \\
T4 v T2 stage & 1.19 & $0.32-4.40$ & \\
T4 $v$ T3 stage & 1.38 & $0.51-3.76$ & \\
N stage & 1.49 & $0.74-3.01$ & 0.269 \\
M stage & 0.42 & $0.24-0.74$ & 0.002 \\
Physical function & $0.88 \ddagger$ & $0.82-0.96$ & 0.002 \\
\hline
\end{tabular}

$\star$ Hazard ratio provides the likelihood of death at any given time for a patient aged 10 years older than another patient.

† Statistical significance of main effect of $T$ stage on survival (2 degrees of freedom)

¥Hazard ratio provides the likelihood of death at any given time for a patient with a physical function score of 10 points more than another patient.

illustrated in table 4 . For a 10 point increase in physical function score there was a $12 \%$ reduction in the likelihood of death at any given time (hazard ratio $0.88,95 \%$ confidence intervals 0.82-0.96). Adjusting for sex, tumour type, and ASA grade did not alter the findings, and hence are not presented here. As perhaps expected (in light of the information used to inform treatment decision), when adjusting for treatment group, a similar influence on the model was seen as with age and $M$ stage. Treatment was not included in the final model.

Median QL scores available for patients alive six months after treatment are shown in table 2 .
Multivariable analyses, adjusting for age, and $\mathrm{T}, \mathrm{N}$, and $\mathrm{M}$ stage, considering the change in QL variables between baseline and six month assessments among the 38 patients alive, found that improvements in emotional function were significantly associated with longer survival $(p<0.0001)$ (table 5). Adjusting for other possible prognostic factors, as described above, did not alter this finding.

\section{Discussion}

This prospective study provides some evidence to support a relationship between patient rated scores of QL and survival. Better baseline physical function was significantly related to longer survival after adjusting for established prognostic factors. In the second hypothesis generating analysis, an improvement in emotional function six months after treatment was significantly related to longer survival after adjusting for established prognostic factors. These data show that QL scores may carry prognostic information for patients with oesophageal cancer over and above other recorded factors. This study was small however and a more highly powered study using appropriate QL tools and clinical data is needed to demonstrate stronger associations between QL scores and survival.

Several different aspects of QL have been associated with survival in a variety of malignancies. ${ }^{1-6}{ }^{17-19}$ High scores for physical, emotional, and social function, and few problems with appetite loss have been reported to independently predict a better outcome. It is difficult to generalise from these studies however because different QL instruments have been used in different cancer sites. In patients with carcinoma of the oesophagus treated with radiotherapy, scores from the Karnofsky performance scale may have prognostic value. ${ }^{18} 19$ Although the Karnofsky performance scale has been used widely in oncology, it is generally considered that this unidimensional tool, completed by a observer, has been superseded by multidimensional instruments that are completed by the patients themselves. The EORTC QLQ-C30 was chosen for this research because many studies have confirmed its validity and it is easily completed by patients. ${ }^{101113}$ Addition of a site specific module, the EORTC QLQ-OES24, for oesophageal cancer may yield more prognostic data because the module addresses QL issues of particular relevance to this group of patients. ${ }^{12}$

The association observed in this study between physical function and survival does not establish a causative relationship between the two since physical function may be a surrogate marker of an unrecognised biological prognostic factor. For instance, patients with micrometastatic disease may have poor physical function and it may be hypothesised that they perceive this (and report it in terms of QL) before it is clinically or radiologically apparent. The association demonstrated in this study between a decrease in emotional function six months after treatment and decreased survival has been hypothesised in several other papers. 
Table 5 Multivariable analyses of median changes in quality of life (QL) scores, $n=38$. The final model

\begin{tabular}{lclc}
\hline Variable & Hazard ratio & $95 \%$ CI & p Value \\
\hline Age & $1.37^{\star}$ & 0.98 to 1.91 & 0.067 \\
T3 $v$ T2 stage & 2.70 & 0.61 to 11.88 & $0.152 \dagger$ \\
T4 $v$ T2 stage & 13.68 & 0.92 to 204.49 & \\
T4 $v$ T3 stage & 5.07 & 0.49 to 52.92 & \\
N stage & 0.32 & 0.11 to 0.92 & 0.035 \\
M stage & 1.90 & 0.08 to 47.72 & 0.697 \\
Emotional function & $0.65 \ddagger$ & 0.52 to 0.81 & $<0.0001$ \\
\hline
\end{tabular}

${ }^{\star}$ Hazard ratio provides the likelihood of death at any given time for a patient aged 10 years older than another patient.

tStatistical significance of main effect of $\mathrm{T}$ stage on survival ( 2 degrees of freedom).

$\ddagger$ Hazard ratio provides the likelihood of death at any given time for a patient with a physical function score of 10 points more than another patient.

Recent data from patients with early breast cancer suggested that high depression and helplessness scores within a year of diagnosis have detrimental effects on survival and event free survival. ${ }^{17}$ This study however does not fully discuss the relevant clinical prognostic factors (such as disease stage), and therefore may be subject to criticism. It is currently not known whether psychological response to cancer influences survival. Emotional function may be a surrogate marker of a presently unrecognised biological indicator of poor outcome.

The sample size in the present study was inadequate and the data can only be used as a hypothesis generating exercise. Whether associations between QL scores and survival are clinically useful will only be demonstrated if deliberate interventions aimed at improving QL are shown to enhance survival.

An earlier version of this paper was presented to the Association of Upper Gastrointestinal Surgeons Meeting, September 1999 at the Royal College of Surgeons of England, and published in abstract form (Br ₹ Surg 2000;87:365).

We thank Mr K Jeyasingham and Mr CP Forrester-Wood for allowing us to study patients in their care.

1 Coates A, Porzsolt F, Osoba D. Quality of life in oncology practise: prognostic value of EORTC QLQ-C30 scores in patients with advanced malignancy. Eur $f$ Cancer 1997;33:1025-30.
2 Dancey J, Dee B, Osoba D, et al. Quality of life scores; an independent prognostic variable in a general population of cancer patients receiving variable. Qual Life Res 1997;6: $151-8$.

3 Montazeri A, Milroy R, Hole D, et al. Quality of life in lung cancer patients. As an important prognostic factor. Lung Cancer 2001;31:233-40.

4 Ganz PA, Lee JJ, Siau J. Quality of life assessment. An independent prognostic variable for survival in lung cancer. Cancer 1991;67:3131-5.

5 Coates AS, Gebski V, Signorini D, et al. Prognostic value of quality of life scores during chemotherapy for advanced quality of life scores during chemotherapy
breast cancer. F Clin Oncol 1992;10:1833-8.

6 Coates AS, Thomson D, McLeod GR, et al. Prognostic value of quality of life scores in a trial of chemotherapy with or without interferon in patients with metastatic melanoma. Eur f Cancer 1993;29A: 1731-4.

7 Kramer JA, Curran D, Piccart M, et al. Identification and interpretation of clinical and quality of life prognostic factors for survival and response to treatment in first-line chemotherapy in advanced breast cancer. Eur $\mathcal{F}$ Cancer 2000;36:1498-506.

8 O'Hanlon D, Harkin M, Daya K, et al. Quality of life assessment in patients undergoing treatment for oesophageal carcinoma. Br F Surg 1995;82:1682-5.

9 Farndon MA, Wayman J, Clague MB, et al. Costeffectiveness in the management of patients with oesophageal cancer. Br F Surg 1998;85:1394-8.

10 Zieren HU, Jacobi CA, Zieren J, et al. Quality of life following resection of oesophageal carcinoma. Brf Surg 1996;83: $1772-5$.

11 Aaronson NK, Ahmedzai S, Bergman B, et al. The European Organization for Research and Treatment of Cancer QLQ-C30; a quality of life instrument for use in international clinical trials in oncology. 7 Natl Cancer Inst international clinical

12 Blazeby JM, Alderson D, Winstone K, et al. Development of a EORTC questionnaire module to be used in quality of life assessment for patients with oesophageal cancer. Eur $\mathcal{F}$ Cancer 1996;32:1912-17.

13 Blazeby JM, Williams MH, Brookes ST, et al. Quality of life measurement in patients with oesophageal cancer. Gut 1995;37:505-8.

14 Fayers P, Aaronson NK, Bjordal K, et al. EORTC QLQ-C30 Scoring Manual. Brussels: Quality of Life Unit, EORTC Data Centre, 1995.

15 Cox DR. Regression models and life-tables. 7 R Stat Soc 1972;34:187-220.

16 Blazeby JM, Farndon JR, Donovan J, et al. A prospective study examining the quality of life of patients with esophageal carcinoma. Cancer 2000;88:1781-7.

17 Watson M, Haviland JS, Greer S, et al. Influence of psychological response on survival in breast cancer; a population based cohort study. Lancet 1999;354:1331-6.

18 Coia LR, Minsky BD, Berkey BA et al. Outcome of patients receiving radiation for cancer of the esophagus; Results of the 1992-1994 patterns of care study. F Clin Oncol 2000;18:455-62.

19 Albertsson M, Ewers SB, Widmark H, et al. Evaluation of the palliative effect of radiotherapy for oesophageal the palliative effect of radiotherapy
carcinoma. Acta Oncol 1989;28:267-70.

20 Hermanek P, Sobin LH. Digestive system tumours. In: Hermanek P, Sobin LH, eds. UICC TNM Classification of Malignant Tumours. Berlin: Springer-Verlag, 1992. 